\title{
The skeletal effects of pioglitazone in type 2 diabetes or impaired glucose tolerance: a randomized controlled trial
}

\author{
Andrew Grey, Mark Bolland, Sheryl Fenwick, Anne Horne, Greg Gamble, \\ Paul L Drury' ${ }^{1}$ and lan R Reid
}

Department of Medicine, University of Auckland, Private Bag 92019, Auckland, New Zealand and ${ }^{1}$ Auckland Diabetes Clinic, Greenlane Clinical Centre, Auckland District Health Board, Auckland 1051, New Zealand
Correspondence should be addressed to A Grey

Email

a.grey@auckland.ac.nz

\begin{abstract}
Objective: Preclinical studies, observational studies, and clinical trials suggest that thiazolidinediones (TZDs) reduce bone mineral density (BMD) and increase fracture risk. Most of the evidence on the skeletal effects of TZDs is from studies of rosiglitazone. We set out to investigate the magnitude and etiology of the adverse skeletal effects of pioglitazone. Design: Double-blind, randomized controlled trial.

Trial registration: Australia New Zealand Clinical Trials Registry, actr.org.au Identifier: ACTRN12607000610437, date of registration 28/11/07.

Methods: A total of 86 people with type 2 diabetes mellitus (T2DM) or impaired glucose tolerance (IGT), median age 64 years, were randomized to receive either pioglitazone $30 \mathrm{mg} /$ day or placebo for 1 year, in addition to their usual diabetes treatments. The primary outcome was change in lumbar spine BMD; secondary outcomes included changes in BMD at other sites and in biochemical markers of bone turnover.

Results: Change in spine BMD was not altered by treatment with pioglitazone $\left(P_{\text {treatment } \times \text { time }}=0.5\right)$. After 1 year, the mean $(95 \% \mathrm{Cl})$ between-groups difference in lumbar spine BMD was $-0.7 \%(-2.1,0.7)$. Pioglitazone increased bone loss at the proximal femur $\left(P_{\text {treatment } \times \text { time }}=0.03\right)$. After 12 months, the between-groups difference in total hip BMD was $-1.2 \%$ $(-2.1,0.2)$. Pioglitazone did not alter change in BMD at other skeletal sites, nor did it affect changes in the levels of either of the biochemical markers of bone turnover, procollagen type $1 \mathrm{~N}$-terminal propeptide, or $\beta$-C-terminal telopeptide of type 1 collagen.

Conclusions: Over 1 year, treatment with pioglitazone $30 \mathrm{mg} /$ day did not produce consistent effects on either BMD or bone turnover in people with T2DM or IGT. The mechanism(s) by which pioglitazone increases fracture risk in T2DM is unclear.
\end{abstract}

\section{Introduction}

Thiazolidinediones (TZDs) are insulin-sensitizing agents used in the management of type 2 diabetes mellitus (T2DM) (1). They are agonists of the $\gamma$-isoform of the peroxisome proliferator-activated receptor (PPAR) family of nuclear transcription factors. In patients with T2DM, TZDs improve glycemic control (1); in patients with impaired glucose tolerance (IGT), they slow the development of T2DM (2).
European Journal of Endocrinology

(2014) 170, 255-262 
Fractures, captured as adverse events in randomized clinical trials, are increased in women with diabetes randomized to TZD therapy compared with those allocated to either placebo or other oral hypoglycemic agents (4). Observational studies reported a similar effect in men with diabetes $(5,6)$. In clinical trials, the excess of fractures was apparent in the appendicular skeleton (7); observational studies in older and larger populations suggested an increase in risk of hip and spine fractures also $(5,8)$.

Although it seems clear that TZDs adversely affect skeletal health, several uncertainties remain. The mechanism(s) by which TZDs increase skeletal fragility in humans is not clear. Short-term studies on healthy older women (9), on younger women with polycystic ovarian syndrome (10), on women with T2DM (11), on men with T2DM (12), and on men with HIV infection (13) suggested that TZDs decrease bone formation and accelerate bone loss. However, other studies have reported increases in markers of bone resorption in participants randomized to TZD therapy (14). Several studies that reported data on markers of bone turnover have been active comparator trials, in which significant changes from baseline in bone markers have also been observed in the non-TZD arm(s). In addition, limited data are available on the magnitude and time course of the effects of TZDs on the human skeleton, and few data are available on the effects of TZDs on appendicular bone mass. Almost all of the clinical data on TZDs and bone health are from studies of rosiglitazone, a medication that has now been withdrawn from use in some jurisdictions because of its adverse effects on vascular health (15).

Several studies have suggested that pioglitazone does not induce the adverse cardiovascular effects that are observed with rosiglitazone (16). Consequently, pioglitazone use remains substantial (17), despite evidence for increased fracture risk (4) and concerns about increased risk of bladder cancer (18). Thus, studying the skeletal effects of TZDs, and specifically of pioglitazone, remains relevant. In this paper, we report the results of a 1-year randomized controlled trial of the effects of pioglitazone on bone mineral density (BMD) and biochemical markers of bone turnover in people with T2DM or IGT.

\section{Subjects and methods}

\section{Protocol}

All visits took place at a clinical research facility. Participants were recruited by newspaper advertisement, by sending written invitation from primary care clinics or by direct invitation from a secondary care clinic. Eligible participants were $>30$ years with T2DM, defined by a fasting blood glucose $>7 \mathrm{mmol} / \mathrm{l}$ and/or serum glucose $>11 \mathrm{mmol} / \mathrm{l} 2 \mathrm{~h}$ after ingesting $75 \mathrm{~g}$ oral glucose, or IGT, defined by fasting blood glucose $6-7 \mathrm{mmol} / \mathrm{l}$ and/or serum glucose $7.8-11 \mathrm{mmol} / 12 \mathrm{~h}$ after ingesting $75 \mathrm{~g}$ oral glucose. Exclusion criteria were current TZD use; congestive heart failure New York Heart Association grade 2 or higher; advanced renal dysfunction (estimated glomerular filtration rate (eGFR) $<30 \mathrm{ml} / \mathrm{min}$ ); clinical liver disease; current malignancy; body weight $>120 \mathrm{~kg}$; previous vertebral, hip, forearm, or humerus fracture; BMD $T$-score at dual total hip or L1-L4 $<-2$; serum $25 \mathrm{OHD}$ $<30 \mathrm{nmol} / \mathrm{l}$; other metabolic bone disease; current use of medications known to influence bone metabolism; and previous use of an aminobisphosphonate. Participants receiving insulin needed to have had a stable daily dose for the 3 months preceding enrolment. Recruitment occurred between October 2008 and April 2011. The flow of participants through the trial is shown in Fig. 1. Among the 86 randomized participants, five withdrew from the study, three in the pioglitazone group (all personal reasons), and two in the placebo group (one personal reasons and one incorrect diagnosis of T2DM). Thirteen participants (eight pioglitazone and five placebo) discontinued study medication during the trial but completed the protocol. The final 1-year study visit occurred in April 2012.

Management of each participant's diabetes during the trial was undertaken by the usual health care provider,

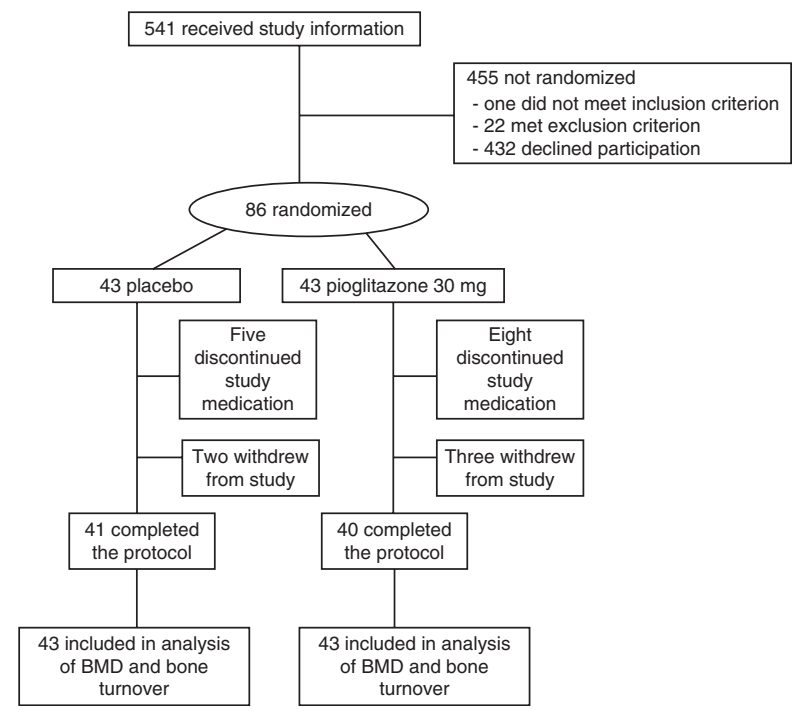

Figure 1

Flow of participants through the study. 
who was informed of their patient's participation. Enquiry was made at each study visit as to the occurrence of dyspnea or peripheral edema. Any participant who reported either symptom was assessed for congestive heart failure by clinical examination and echocardiography. If congestive heart failure was diagnosed, study medication was discontinued.

\section{Randomization and masking}

The pioglitazone and placebo tablets differed in appearance, so to ensure double blinding they were placed in identical opaque capsules by the research staff members who had no contact with trial participants or involvement in study procedures. The participants were randomly allocated to receive a daily capsule containing pioglitazone or placebo for 12 months. For participants allocated to pioglitazone, the dose was $15 \mathrm{mg}$ daily for the first month, and thereafter $30 \mathrm{mg}$ daily. No other study medication was administered. The treatment allocations were randomized by the study statistician, using a variable block size schedule, based on computer-generated (Excel 2010) random numbers. Only the statistician had access to treatment allocation codes, and he had no contact with study participants. All the other study personnel and subjects were blinded to treatment allocation throughout. Only the study statistician saw unblinded data. Success of blinding was assessed by asking participants at the end of the trial whether they had been allocated to active or placebo therapy, or did not know, and James' Blinding Index was calculated (19).

\section{Outcomes}

The primary endpoint was change in lumbar spine BMD at 1 year. The secondary endpoints included change in BMD at dual total hip, total body, and forearm, and change in the bone turnover markers, serum procollagen type $1 \mathrm{~N}$-terminal propeptide (P1NP) and $\beta$-C-terminal telopeptide of type 1 collagen ( $\beta$-CTX). Assuming a s.D. for the change in the lumbar spine (L1-L4) BMD of 5\%, the study was adequately ( $>80 \%$ ) powered to detect a difference (two-tailed $P<0.05$ ) of $4 \%$ in spine $\mathrm{BMD}$ between the treatment groups.

BMD was measured at baseline, 3, 6, 9, and 12 months at the lumbar spine (L1-L4), dual proximal femur, forearm, and total body using a Lunar Prodigy dual-energy X-ray absorptiometer (GE Lunar, Madison, WI, USA). The coefficients of variation $(\mathrm{CV})$ for the measurement of total hip and lumbar spine BMDs in our laboratory are 1.1 and $1.4 \%$ respectively.

The serum bone turnover markers, P1NP and $\beta$-CTX, were measured in the samples collected after overnight fast at baseline, 1, 3, and 12 months using the Roche Elecsys 2010 platform (Roche Diagnostics). The samples were stored at $-70{ }^{\circ} \mathrm{C}$ until the conclusion of the study, when they were batch-analyzed. The $\mathrm{CV}$ of these markers are $\beta$-CTX, 5.1\% and PINP, $1.9 \%$.

\section{Statistical analysis}

The data were analyzed using procedures of SAS, version 9.2 (SAS Institute, Inc., Cary, NC, USA). All analyses were by intention to treat. Continuous, normally distributed variables were analyzed with a mixed-models ANOVA. The main effects of time and treatment and their interaction (treatment $\times$ time) were included in a fully saturated model, with the absolute level being the dependent variable. In complementary analyses, change from baseline was the dependent variable and the appropriate baseline value included as a covariate. In the event that the interaction term failed to reach statistical significance, a reduced model was fitted to explore the remaining main effects. Significant main or interaction effects were further explored using the method of Tukey to preserve the overall pairwise error rate. The data are presented as mean and 95\% CI unless otherwise indicated. All tests were twotailed, and $P<0.05$ was considered significant.

\section{Role of the funding source}

The study sponsor had no role in study design, in the collection, analysis, and interpretation of data, in the writing of the report, or in the decision to submit the paper for publication.

\section{Trial registration}

The study received ethical approval from the Northern Y Regional Ethics Committee and was registered with the Australian New Zealand Clinical Trials Registry, ACTRN12607000610437, date of registration 28/11/07. All participants gave written, informed consent.

\section{Results}

The baseline characteristics of the 86 study participants are presented in Table 1. All but one participant had T2DM. As expected in a population with T2DM (20), BMD at each 
Table 1 Baseline data for trial participants. Data are mean (s.D.) or $n(\%)$.

\begin{tabular}{l}
\hline \\
\hline$n$ \\
Age (years) \\
Female, $n(\%)$ \\
Weight $(\mathrm{kg})$ \\
Height $(\mathrm{cm})$ \\
BMI $\left(\mathrm{kg} / \mathrm{m}^{2}\right)$ \\
HbA1c, $\%(\mathrm{mmol} / \mathrm{mol})$ \\
\\
Insulin therapy, $n(\%)$ \\
Other oral hypoglycemic, $n(\%)$ \\
Current smoker $n(\%)$ \\
Calcium intake (mg/day) \\
Serum $25 O H D(\mathrm{nmol} / \mathrm{l})$ \\
Fractures during adulthood, \\
$\quad n(\%)$ \\
Lumbar spine BMD $\left(\mathrm{g} / \mathrm{cm}^{2}\right)$ \\
$T$-score \\
Total hip BMD $\left(\mathrm{g} / \mathrm{cm}^{2}\right)$ \\
$T$-score \\
Total body BMD $\left(\mathrm{g} / \mathrm{cm}^{2}\right)$ \\
$T$-score \\
P1NP ( $\mu$ g/l) \\
$\beta$-CTX (ng/l)
\end{tabular}

\begin{tabular}{ccc}
\hline \multicolumn{1}{c}{ Placebo } & & Pioglitazone \\
\cline { 1 - 1 } 43 & & 43 \\
$63(38-84)$ & & $64(48-79)$ \\
$20(47)$ & & $22(51)$ \\
$86(15)$ & & $85(16)$ \\
$167(9)$ & & $166(10)$ \\
$31(5)$ & & $31(5)$ \\
$7.5(3.2)$ & & $7.4(3.5)$ \\
$58(11)$ & & $57(15)$ \\
$12(28)$ & & $5(12)$ \\
$37(86)$ & & $33(77)$ \\
$5(12)$ & & $1(2)$ \\
$850(450)$ & & $760(380)$ \\
$68(25)$ & & $63(24)$ \\
$2(5)$ & & $2(5)$ \\
& & \\
$1.36(0.24)$ & & $1.30(0.18)$ \\
$1.3(2.0)$ & & $0.8(1.5)$ \\
$1.11(0.12)$ & & $1.11(0.15)$ \\
$0.4(1.0)$ & $0.4(1.0)$ \\
$1.25(0.10)$ & $1.24(0.11)$ \\
$1.0(1.2)$ & $0.9(1.1)$ \\
$35(15)$ & $38(22)$ \\
$240(120)$ & $260(130)$ \\
& &
\end{tabular}

Serum 25OHD, serum 25-hydroxyvitamin $D$; $B M D$, bone mineral density; $\mathrm{P} 1 \mathrm{NP}$, procollagen type $1 \mathrm{~N}$-terminal propeptide; $\beta$-CTX, $\beta$-C-terminal telopeptide of type 1 collagen.

${ }^{\mathrm{a}}$ Data presented as median (range)

site was robust. During the trial, HbA1c decreased in the pioglitazone group (change from baseline $-0.4 \%(-0.6$, $-0.1) ;-3.6 \mathrm{mmol} / \mathrm{mol}(-6.1,-1.1)$ ) while that in the control group was unchanged (change from baseline $0.1 \%$ $(-0.1,0.3) ; 0.8 \mathrm{mmol} / \mathrm{mol}(-1.5,3.0))(P=0.01$ pioglitazone vs placebo). Mean compliance did not differ between groups (placebo $87 \%$, pioglitazone $85 \%, P=0.7$ ). At the end of the trial, a majority of participants in each group (56\% placebo and 61\% pioglitazone) stated that they did not know their treatment allocation. James' Blinding Index was 0.6 , indicating moderate success in achieving blinding (19).

\section{Bone mineral density}

Figure 2 shows the BMD data at lumbar spine and dual total hip. Pioglitazone did not alter BMD at the lumbar spine $\left(P_{\text {treatment } \times \text { time }}=0.5\right)$. After 12 months, the betweengroups difference in lumbar spine BMD was $-0.7 \%(-2.1$, 0.7). Pioglitazone increased bone loss at the proximal femur $\left(P_{\text {treatment } \times \text { time }}=0.03\right)$. After 12 months, the between-groups difference in total hip BMD was $-1.2 \%$ $(-2.1,0.2)$.
Figure 3 shows the BMD data at the 33\% (proximal) forearm and total body sites, which are wholly or predominantly composed of cortical bone. At neither site was there evidence that pioglitazone induced bone loss. After 12 months, the between-groups difference in proximal forearm BMD was $-0.9 \%(-2.2,0.3)$ and $-0.5 \%(-1.2,0.1)$ at the total body.

Post hoc analyses did not suggest a differential effect of pioglitazone on BMD by gender. Mean between-groups differences in BMD in female participants after 12 months ranged from $-0.1 \%$ at the lumbar spine to $-2.0 \%$ at the total hip, while in male participants the mean differences ranged from $-0.3 \%$ at the total body to $-1.2 \%$ at the lumbar spine.
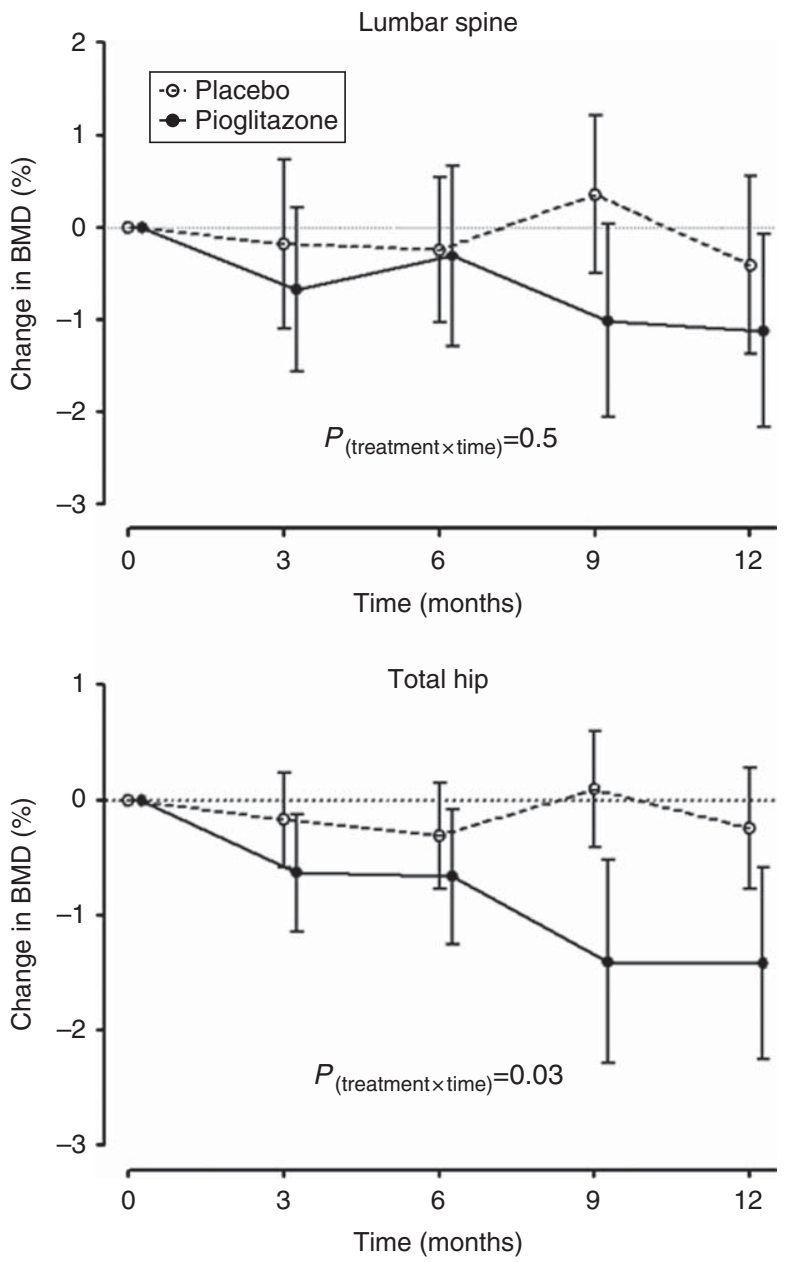

\section{Figure 2}

Effects of pioglitazone on BMD at the lumbar spine L1-L4 (top), and dual total hip (bottom) over 1 year in people with type 2 diabetes mellitus or impaired glucose tolerance. Data are mean $(95 \% \mathrm{Cl})$ percent change from baseline. 

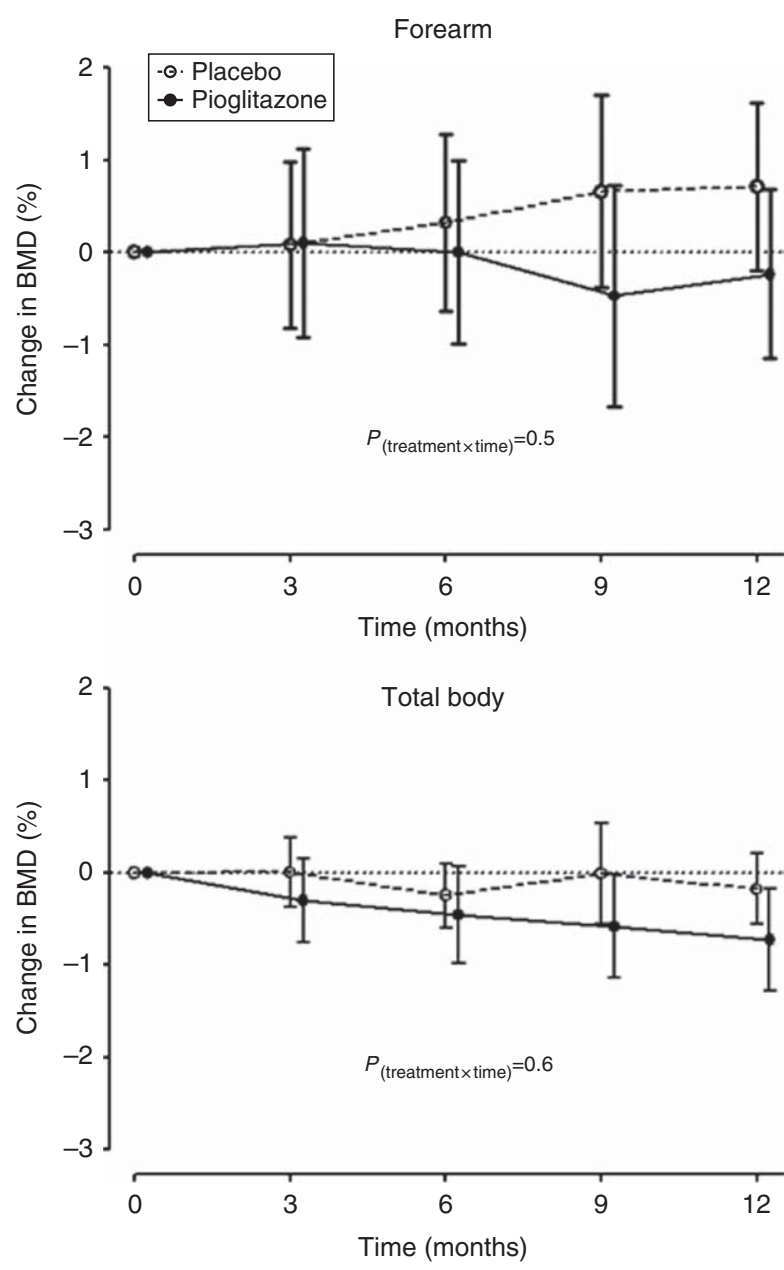

Figure 3

Effects of pioglitazone on BMD at the 33\% forearm (top), and total body (bottom) over 1 year in people with type 2 diabetes mellitus or impaired glucose tolerance. Data are mean $(95 \% \mathrm{Cl})$ percent change from baseline.

Body weight declined by $0.04 \mathrm{~kg}(-1.01,0.93)$ in the control group and increased by $1.4 \mathrm{~kg}(0.20,2.60)$ in the pioglitazone group. Including change in body weight as a covariate did not alter the results $\left(P_{\text {treatment } \times \text { time }}\right.$ for lumbar spine $\mathrm{BMD}=0.5)$. Including age as a covariate in the analyses did not alter the results $\left(P_{\text {treatment } \times \text { time }}\right.$ for lumbar spine $\mathrm{BMD}=0.5$ ).

\section{Bone turnover markers}

Figure 4 shows the results of bone turnover marker measurements. The marker of bone formation, P1NP, was marginally higher during the trial in the pioglitazone group $\left(P_{\text {treatment } \times \text { time }}=0.05\right)$. After 12 months, P1NP was higher by $14 \%(0.3,28)$ in the pioglitazone group. The marker of bone resorption, $\beta$-CTX, was not affected by pioglitazone $\left(P_{\text {treatment } \times \text { time }}=0.6\right)$. After 12 months, $\beta$-CTX was higher by $9 \%(-11,28)$ in the pioglitazone group.

\section{Adverse events}

Lower limb edema occurred more frequently in the pioglitazone group $(n=8)$ than in the placebo group $(n=1)(P=0.02)$. Congestive heart failure was diagnosed in two participants in the pioglitazone group and one
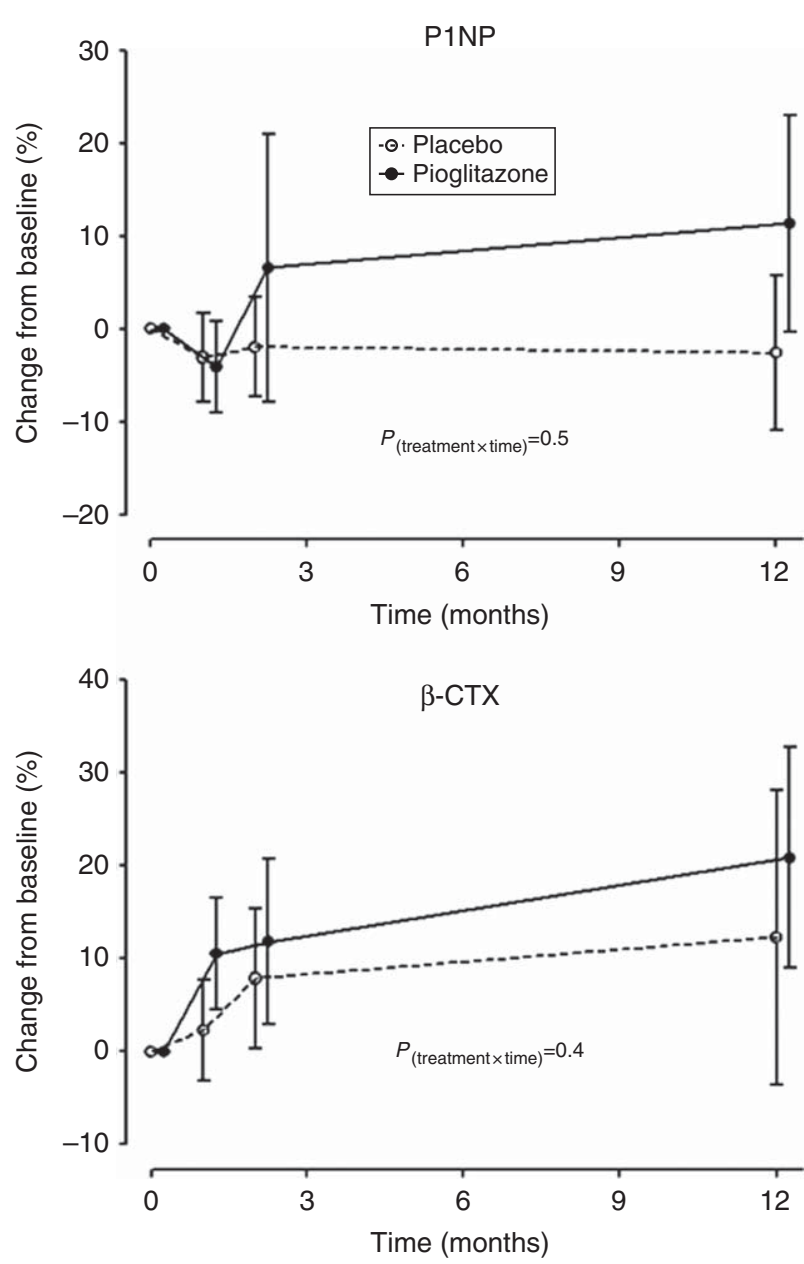

\section{Figure 4}

Effects of pioglitazone on the markers of bone turnover, procollagen type $1 \mathrm{~N}$-terminal propeptide ( $\mathrm{P} 1 \mathrm{NP}$, top), and $\beta$-C-terminal telopeptide of type 1 collagen ( $\beta$-CTX, bottom) over 1 year in people with type 2 diabetes mellitus or impaired glucose tolerance. Data are mean $(95 \% \mathrm{Cl})$ percent change from baseline. 
participant in the placebo group $(P=0.7)$. In the pioglitazone group, study medication was discontinued by eight participants, because of congestive heart failure $(n=2)$, edema without congestive heart failure $(n=4)$, painful feet without edema $(n=1)$, and paraesthesia in the hands $(n=1)$. In the placebo group, study medication was discontinued by five participants, because of congestive heart failure $(n=1)$, musculoskeletal pain $(n=2)$, nausea $(n=1)$, and nonspecific symptoms $(n=1)$. In the pioglitazone group, three fractures (one toe and two ankles) occurred in two participants. No fractures occurred in the placebo group.

\section{Discussion}

This trial was established in response to the findings of an increased incidence of fractures identified by adverse events reporting in pharmaceutical company-sponsored trials of TZDs (4), and of accelerated bone loss in shortterm trials of TZDs in healthy older women (9), women with polycystic ovary syndrome (10), and older women with T2DM (11). Evidence from preclinical studies (3) and from clinical trials implicated both decreased bone formation (9) and increased bone resorption (14) as mechanisms underpinning the acceleration of bone loss. We found that $30 \mathrm{mg} /$ day of pioglitazone did not increase bone loss at the lumbar spine over 12 months in a population of men and women with T2DM. Pioglitazone marginally increased bone loss at the proximal femur, but had no effect on BMD at skeletal sites enriched for cortical bone, the proximal forearm, and total body. Pioglitazone marginally increased levels of the bone formation marker P1NP, but did not affect those of the bone resorption marker, $\beta$-CTX.

Most of the evidence for skeletal harm of TZDs comes from studies of rosiglitazone (3). Although both clinically available TZDs improve insulin sensitivity and glycemic control, there may be differences between the drugs in their effects on other tissues. Thus, rosiglitazone increases the risk of cardiovascular events (21), while pioglitazone may not (16). Pioglitazone may increase the risk of bladder cancer (18), an effect not associated with rosiglitazone (22). We elected to study pioglitazone because at the time the trial was planned there was already some evidence of increased vascular risk from treatment with rosiglitazone, findings which subsequently led to its withdrawal from clinical use in some jurisdictions (15). Our results suggest that pioglitazone, administered at a dose frequently used in clinical practice, has small effects on bone turnover and BMD. They are congruent with a preliminary report from a randomized placebo-controlled trial of $45 \mathrm{mg} /$ day pioglitazone in postmenopausal women with glucose intolerance, in which there was no effect of the TZD on total hip or lumbar spine BMD or markers of bone turnover during 12 months of exposure (23). In addition, a randomized trial of rosiglitazone vs metformin demonstrated modest decrements in BMD (1-2\% in the axial skeleton) and increases in bone turnover ( $20 \%)$ over 12 months in postmenopausal women with T2DM (24). Similarly small decreases in BMD throughout the skeleton (0.2-2.2\%), without alteration in markers of bone turnover, were observed in participants with T2DM in a randomized trial of rosiglitazone and metformin vs metformin, over 18 months of treatment (25).

It is therefore apparent that $12-18$ months of treatment of people with T2DM with a TZD produces small adverse effects on surrogate markers for skeletal health. The changes are similar in magnitude, although opposite in direction, to those observed in response to interventions which cause marginal reductions in fracture risk, such as calcium supplementation (26). An important consideration, therefore, is how TZDs increase fracture risk, a finding that emerged from the analyses of adverse events in trials of both rosiglitazone and pioglitazone (4). In those analyses, the relative risk of fracture was increased by 1.5- to 2-fold and appeared to be restricted to the appendicular skeleton and to women $(4,27,28)$. Subsequent observational studies reported increased fracture risk after exposure to a TZD in men, and in the axial skeleton (5). A 1.5- to 2-fold increase in fracture risk, if attributable to changes in BMD, would be predicted to be associated with a decrement in BMD approaching 1 s.D. ( 10\%) in size (29).

Although the risk of fracture is consistently reported to be increased in T2DM (30), this observation cannot be explained by alterations in BMD measured by dual-energy x-ray absorptiometry (DXA) (20), in bone structure assessed by computed tomography (31), or in bone metabolism as assessed by turnover markers (30). An increased risk of falling, consequent upon the vascular, visual, and neurological co-morbidities associated with T2DM (32), and/or impaired bone quality secondary to nonenzymatic glycosylation of components of the bone matrix (33), have been invoked to explain skeletal fragility in T2DM. To our knowledge, there is no evidence that TZDs increase risk of falls. TZDs reduce glucose levels, so would be predicted to reduce nonenzymatic glycosylation. However, it remains possible that TZDs might increase fracture risk by adversely affecting bone quality in a manner that is not captured by current assessment modalities. 
Although our trial was randomized, placebocontrolled, and double-blind, it had some limitations. The trial was powered to detect a between-groups difference in spine $\mathrm{BMD}$ of $4 \%$, a change that was plausible based on data from short-term trials (9), and that over 1 year that would clearly be clinically and biologically significant. The study duration was only 12 months; it is possible that a longer duration of treatment might reveal a greater cumulative effect of pioglitazone on bone metabolism. If this was so, the drug might produce clinically important decrements in BMD during long-term therapy of T2DM. However, the increased risk of fracture in an active comparator trial of rosiglitazone became apparent during the second year (27), suggesting that, if alterations in either BMD or bone turnover were contributory, they would probably have been apparent by the end of 1 year. Finally, our trial had limited power to detect differences in the skeletal effects of pioglitazone by gender.

In summary, the results of this trial suggest that 1 year of treatment of people with T2DM or IGT with a standard dose of pioglitazone did not produce clinically important changes in either BMD or biochemical markers of bone turnover. Our findings are congruent with those from recently published trials of rosiglitazone of $45 \mathrm{mg} / \mathrm{day}$ pioglitazone. Whether there is a cumulative effect of pioglitazone on bone metabolism over longer durations of therapy is not known. The explanation for the increased risk of fractures observed in both randomized trials and observational studies of TZDs remains unclear.

\section{Declaration of interest}

A Grey, M Bolland, S Fenwick, A Horne, and G Gamble have no conflict of interest to declare; $\mathrm{P}$ L Drury has received consultancy fees from Novo-Nordisk; I R Reid has received research funding, speaker, and consultancy fees from Novartis, Merck, and Amgen.

\section{Funding}

Grant support from the Health Research Council of New Zealand.

\section{Author contribution statement}

A Grey designed the study and drafted the manuscript; M Bolland designed the study; S Fenwick recruited the participants and collected the data; A Horne recruited the participants and collected the data; G Gamble designed the study and performed the statistical analyses; P L Drury designed the study; and I R Reid designed the study. All authors reviewed and edited the draft manuscript. A Grey is the guarantor of this work and, as such, had full access to all the data in the study and takes responsibility for the integrity of the data and the accuracy of the data analysis.
Acknowledgements

The authors thank Tim Cundy for critically reviewing the manuscript.

\section{References}

1 Yki-Jarvinen H. Thiazolidinediones. New England Journal of Medicine 2004351 1106-1118. (doi:10.1056/NEJMra041001)

2 The Dream Trial Investigators. Effect of rosiglitazone on the frequency of diabetes in patients with impaired glucose tolerance or impaired fasting glucose: a randomised controlled trial. Lancet $2006 \mathbf{3 6 8}$ 1096-1105. (doi:10.1016/S0140-6736(06)69420-8)

3 Grey AB. Thiazolidinedione-induced skeletal fragility - mechanisms and implications. Diabetes, Obesity \& Metabolism 200911 275-284. (doi:10.1111/j.1463-1326.2008.00931.x)

4 Loke YK, Singh S \& Furberg CD. Long-term use of thiazolidinediones and fractures in type 2 diabetes: a meta-analysis. CMAJ: Canadian Medical Association Journal 2009180 32-39. (doi:10.1503/cmaj.080486)

5 Douglas IJ, Evans SJ, Pocock S \& Smeeth L. The risk of fractures associated with thiazolidinediones: a self-controlled case-series study. PLoS Medicine 20096 e1000154. (doi:10.1371/journal.pmed.1000154)

6 Meier C, Kraenzlin ME, Bodmer M, Jick SS, Jick H \& Meier CR. Use of thiazolidinediones and fracture risk. Archives of Internal Medicine 2008 168 820-825. (doi:10.1001/archinte.168.8.820)

7 Kahn SE, Haffner SM, Heise MA, Herman WH, Holman RR, Jones NP, Kravitz BG, Lachin JM, O'Neill MC, Zinman B et al. Glycemic durability of rosiglitazone, metformin, or glyburide monotherapy. New England Journal of Medicine 2006355 2427-2443. (doi:10.1056/NEJMoa066224)

8 Solomon DH, Cadarette SM, Choudhry NK, Canning C, Levin R \& Sturmer T. A cohort study of thiazolidinediones and fractures in older adults with diabetes. Journal of Clinical Endocrinology and Metabolism 200994 2792-2798. (doi:10.1210/jc.2008-2157)

9 Grey A, Bolland M, Gamble G, Wattie D, Horne A, Davidson J \& Reid IR. The peroxisome proliferator-activated receptor- $\gamma$ agonist rosiglitazone decreases bone formation and bone mineral density in healthy postmenopausal women: a randomized, controlled trial. Journal of Clinical Endocrinology and Metabolism 200792 1305-1310. (doi:10.1210/ jc.2006-2646)

10 Glintborg D, Andersen M, Hagen C, Heickendorff L \& Hermann AP. Association of pioglitazone treatment with decreased bone mineral density in obese premenopausal patients with polycystic ovary syndrome: a randomized, placebo-controlled trial. Journal of Clinical Endocrinology and Metabolism 200893 1696-1701. (doi:10.1210/jc. 2007-2249)

11 Berberoglu Z, Yazici AC \& Demirag NG. Effects of rosiglitazone on bone mineral density and remodelling parameters in postmenopausal diabetic women: a 2-year follow-up study. Clinical Endocrinology 2010 73 305-312. (doi:10.1111/j.1365-2265.2010.03784.x)

12 Yaturu S, Bryant B \& Jain SK. Thiazolidinedione treatment decreases bone mineral density in type 2 diabetic men. Diabetes Care 200730 1574-1576. (doi:10.2337/dc06-2606)

13 Ross AC, Hileman CO, Brown TT, Fedarko N, Storer N, Labbato D \& McComsey GA. Bone effects of rosiglitazone in HIV-infected patients with lipoatrophy. HIV Clinical Trials 201213 212-221. (doi:10.1310/ hct1304-212)

14 Zinman B, Haffner SM, Herman WH, Holman RR, Lachin JM, Kravitz BG, Paul G, Jones NP, Aftring RP, Viberti G et al. Effect of rosiglitazone, metformin, and glyburide on bone biomarkers in patients with type 2 diabetes. Journal of Clinical Endocrinology and Metabolism 201095 134-142. (doi:10.1210/jc.2009-0572)

15 European Medicines Agency (http://www.ema.europa.eu/ema/index. jsp?curl=pages/news_and_events/news/2010/09/news_detail_001119. jsp\&mid=WC0b01ac058004d5c1, 2010). Accessed 26 April 2013.

16 Lincoff AM, Wolski K, Nicholls SJ \& Nissen SE. Pioglitazone and risk of cardiovascular events in patients with type 2 diabetes mellitus: 
a meta-analysis of randomized trials. Journal of Clinical Endocrinology and Metabolism 2007298 1180-1188.

17 Cohen A, Rabbani A, Shah N \& Alexander GC. Changes in glitazone use among office-based physicians in the U.S., 2003-2009. Diabetes Care 201033 823-825. (doi:10.2337/dc09-1834)

18 Hillaire-Buys D \& Faillie J-L. Pioglitazone and the risk of bladder cancer. BMJ 2012344 e3500. (doi:10.1136/bmj.e3500)

19 James KE, Bloch DA, Lee KK, Kraemer HC \& Fuller RK. An index for assessing blindness in a multi-centre clinical trial: disulfiram for alcohol cessation: a VA cooperative study. Statistics in Medicine 199615 1421-1434. (doi:10.1002/(SICI)1097-0258(19960715)15:13<1421:: AID-SIM266 > 3.0.CO;2-H)

20 Vestergaard P. Discrepancies in bone mineral density and fracture risk in patients with type 1 and type 2 diabetes - a meta-analysis. Osteoporosis International 200718 427-444. (doi:10.1007/s00198-006-0253-4)

21 Nissen SE \& Wolski K. Rosiglitazone revisited: an updated meta-analysis of risk for myocardial infarction and cardiovascular mortality. Archives of Internal Medicine 2010170 1191-1201. (doi:10.1001/archinternmed. 2010.207)

22 Tseng C-H. Rosiglitazone is not associated with an increased risk of bladder cancer. Cancer Epidemiology 201337 385-389. (doi:10.1016/ j.canep.2013.03.013)

23 Bone HG, Lindsay R, McClung MR, Perez AT, Raanan MG \& Spanheimer RG. Effects of pioglitazone on bone in postmenopausal women with impaired fasting glucose or impaired glucose tolerance: a randomized, double-blind, placebo-controlled study. Journal of Clinical Endocrinology and Metabolism 2014. In press. (doi:10.1210/ jc.2012-4096)

24 Bilezikian JP, Josse RG, Eastell R, Lewiecki EM, Miller CG, Wooddell M, Northcutt AR, Kravitz BG, Paul G, Cobitz AR et al. Rosiglitazone decreases bone mineral density and increases bone turnover in postmenopausal women with type 2 diabetes mellitus. Journal of Clinical Endocrinology and Metabolism 201398 1519-1528. (doi:10.1210/ jc.2012-4018)

25 Borges JL, Bilezikian JP, Jones-Leone AR, Acusta AP, Ambery PD, Nino AJ, Grosse M, Fitzpatrick LA \& Cobitz AR. A randomized, parallel group, double-blind, multicentre study comparing the efficacy and safety of Avandamet (rosiglitazone/metformin) and metformin on long-term glycaemic control and bone mineral density after 80 weeks of treatment in drug-naive type 2 diabetes mellitus patients. Diabetes, Obesity \& Metabolism 201113 1036-1046. (doi:10.1111/j.1463-1326. 2011.01461.x)

26 Tang BM, Eslick GD, Nowson C, Smith C \& Bensoussan A. Use of calcium or calcium in combination with vitamin D supplementation to prevent fractures and bone loss in people aged 50 years and older: a meta-analysis. Lancet 2007370 657-666. (doi:10.1016/S0140-6736 (07)61342-7)

27 Kahn SE, Zinman B, Lachin JM, Haffner SM, Herman WH, Holman RR, Kravitz BG, Yu DH, Heise MA, Aftring RP et al. Rosiglitazone-associated fractures in type 2 diabetes - an analysis from a diabetes outcome progression trial (ADOPT). Diabetes Care 200831 845-851. (doi:10.2337/dc07-2270)

28 US Food and Drug Administration (http://www.fda.gov/Safety/ MedWatch/SafetyInformation/SafetyAlertsforHumanMedicalProducts/ ucm150451.htm, 2007). Accessed 19 April 2013.

29 Marshall D, Johnell O \& Wedel H. Meta-analysis of how well measures of bone mineral density predict occurrence of osteoporotic fractures. BMJ 1996312 1254-1259. (doi:10.1136/bmj.312.7041.1254)

30 Hamann C, Kirschner S, Gunther K-P \& Hofbauer LC. Bone, sweet bone - osteoporotic fractures in diabetes mellitus. Nature Reviews. Endocrinology 20128 297-305. (doi:10.1038/nrendo.2011.233)

31 Melton LJ III, Riggs BL, Leibson CL, Achenbach SJ, Camp JJ, Bouxsein ML, Atkinson EJ, Robb RA \& Khosla S. A bone structural basis for fracture risk in diabetes. Journal of Clinical Endocrinology and Metabolism 200893 4804-4809. (doi:10.1210/jc.2008-0639)

32 Schwartz AV, Hillier TA, Sellmeyer DE, Resnick HE, Gregg E, Ensrud KE, Schreiner PJ, Margolis KL, Cauley JA, Nevitt MC et al. Older women with diabetes have a higher risk of falls: a prospective study. Diabetes Care 200225 1749-1754. (doi:10.2337/diacare.25.10.1749)

33 Leslie WD, Rubin MR, Schwartz AV \& Kanis JA. Type 2 diabetes and bone. Journal of Bone and Mineral Research 201227 2231-2237. (doi:10.1002/jbmr.1759)

Received 29 September 2013

Revised version received 4 November 2013

Accepted 11 November 2013 